\title{
Reimbursement Approaches for Radiopharmaceutical Dosimetry: Current Status and Future Opportunities
}

\author{
Stephen A. Graves ${ }^{1}$, Alexandru Bageac ${ }^{2}$, James R. Crowley ${ }^{3}$, and Denise A.M. Merlino ${ }^{4}$ \\ ${ }^{1}$ Department of Radiology, University of Iowa, Iowa City, Iowa; ${ }^{2}$ Radiology Associates of North Texas, Fort Worth, Texas; ${ }^{3}$ Diagnostic \\ Radiology, Carilion Clinic, Roanoke, Virginia; and ${ }^{4}$ Merlino Healthcare Consulting Corp., Magnolia, Massachusetts
}

\begin{abstract}
Interest in performing dosimetry for clinical radiopharmaceutical therapy procedures has grown in recent years. Several approved therapies include dosimetry in the Food and Drug Administration-approved label instructions, and other therapies are best used under a patienttailored paradigm. This paper, which is a product of the Society of Nuclear Medicine and Molecular Imaging Dosimetry Task Force, presents motivations and general workflows for radiopharmaceutical therapy dosimetry, as well as existing strategies for obtaining reimbursement for clinical activities related to dosimetry. Several specific patient examples are provided, including suggested codes for reimbursement. In addition to current reimbursement approaches, key dosimetry services that are not supported under the current coding structure are presented and suggested as areas of focus in the coming years.
\end{abstract}

Key Words: RPT; dosimetry; SPECT/CT; PET/CT

J Nucl Med 2021; 62:48S-59S

DOI: 10.2967/jnumed.121.262752

$\mathbf{R}$ adiopharmaceutical therapy (RPT) is a rapidly growing oncologic intervention whereby electron- or $\alpha$-emitting radionuclides, formulated for accumulation within or near cancer cells, are administered by intravenous, intraarterial, or interstitial injection. The mechanism for accumulation within or near cancer cells can be physical in nature, such as ${ }^{90} \mathrm{Y}$-labeled microspheres that become trapped in the arterioles of hypervascular lesions, or biochemical in nature, such as the binding of a radiolabeled peptide or antibody to a biologic receptor.

In most cases, the mass of radiolabeled compound administered for therapy is below any threshold for pharmacologic effects, and it is primarily the energy imparted into tissue by radioactive decay that effects a therapeutic response. The interactions between radiation and human biology-including biologic effects - have been extensively investigated over the last approximately125 y $(1,2)$. The primary endpoints of radiation therapy are so-called deterministic effects in target and nontarget tissue. (Stochastic effects, such as secondary hematologic malignancies, have also been shown to result from radiation exposure and chemotherapy. Current models suggest that these effects are not associated with a dose threshold, but rather the effect risk is thought to increase with increasing cumulative treatment. Rather than individualized dosimetry for

Received Jun. 22, 2021; revision accepted Sep. 22, 2021.

For correspondence or reprints, contact Stephen A. Graves (stephen-agraves@uiowa.edu).

COPYRIGHT @ 2021 by the Society of Nuclear Medicine and Molecular Imaging. toxicity avoidance, stochastic effects are better informed by population-level dosimetry data for risk modeling.) Examples of a deterministic effects include radiation-induced nephropathy (kidney damage) and radiation-induced tumor shrinkage. Deterministic effects, which are the product of cell killing, are associated with a dose threshold, below which no effect is observed. Beyond the radiation dose threshold, the severity or magnitude of a deterministic effect is expected to increase with increasing dose. These dose-dependent effects for various biologic endpoints, tissue types, radiation types, and dose rates have been described in literature.

Radiation dose from RPT is therefore a measure that is expected to correlate with tumor control probability and normal-tissue complication probability. Indeed, there is a growing body of evidence showing that dose-response relationships are observed in RPT (3-10). Although not covered in this paper, the current state of knowledge regarding normal-tissue toxicity relationships and dose-response relationships within the context of RPT is thoroughly described in 2 other papers within this dosimetry supplement. Dose to tumors and normal tissues can vary widely among patients for a given administered activity level due to differences in tissue mass, pharmacokinetics, tissue geometries, and tumor phenotype (11-13). It is therefore critical to monitor patient-specific radiation-absorbed dose by established dosimetry techniques, whereby appropriate changes in management may be made. As with other types of radiation therapy, applying these therapies under a dosimetry-guided paradigm allows clinicians to minimize the risk of long-term toxic side effects, as well as assess for potential benefit in a particular patient.

\section{DOSIMETRY FOR RPT}

The process of obtaining patient-specific dosimetry for RPT involves characterizing the time-ordered distribution of radiopharmaceutical in the body, especially those tissues that are receiving the greatest radiation dose, or those that are naturally most sensitive to radiation. Techniques currently available for obtaining data regarding the distribution of radiopharmaceutical in a patient include the following: whole-body (WB) emission counting (1-dimensional projection of $\gamma$-emitting activity in a patient); planar $\gamma$-imaging (2-dimensional projection of $\gamma$-emitting activity in a patient); SPECT imaging (3-dimensional [3D] reconstruction of $\gamma$-emitting activity in a patient); PET imaging (3D reconstruction of $\beta^{+}$-emitting activity in a patient); and blood or urine sampling (average activity concentration in compartment).

Generally, it is not optimal to rely on the use of only one of these technologies independently for patient-specific dosimetry, as each has weaknesses. With that said, the dosimetric accuracy and precision that can be obtained by use of only one of these datacollection techniques may be appropriate depending on the 
particular RPT and specific patient management needs. Additionally, the number of data-collection time points can influence the accuracy of dosimetric calculations, with increased data collection being associated with improved dosimetric precision (11,14-19). Considerations needed when developing a dosimetry plan for a given RPT and patient should include: tissues of interest for dosimetry, potential impact of dosimetry on patient management (thus necessitating a certain level of accuracy and precision), and the ability of a patient to undergo dosimetric data collection. Even within a particular RPT, these factors vary on a per-patient basis, thus necessitating flexibility in dosimetry methods and associated reimbursement mechanisms.

Normal organs receiving the highest levels of absorbed dose in the body tend to be organs that are involved in concentrating and excreting the radiopharmaceutical, such as the liver, kidneys, bladder, and gastrointestinal tract. Significant radiation dose is also commonly observed in the spleen and secretory tissues (salivary glands, adrenal glands, pituitary gland). Although not typically receiving the highest absorbed dose, the bone marrow is a particularly radiosensitive tissue, and one that is of importance in RPT dosimetry. For a given RPT, usually only 1 or 2 of these organs will limit the quantity of radiopharmaceutical that can be administered without exceeding toxicity thresholds. A summary of approved and late-stage investigational agents, and their most commonly limiting normal organ tissues (20-29), is listed in Table 1.

In addition to consideration of dose-limiting normal organs, tumor dosimetry provides valuable information regarding potential patient benefit or the need for modifications to administered activity to reach a certain probability of benefit. Although an extensive review of tumor and normal organ dose-response relationships is beyond the scope of this document, typically solid tumor doses (from low-linear energy transfer sources) in excess of 100 Gy are needed to achieve high rates of response, whereas doses of less than approximately 50 Gy often do not provide therapeutic benefit from RPTs $(7,9,10,30-33)$. In some cases, potential patient benefit may be minimal, thereby leading to a decision to not proceed with therapy. In this situation, unnecessary radiation exposure to the patient and public can be avoided, as well as an overall reduction in health-care costs. On the other hand, if a patient's organ dosimetry is favorable, and tumor targets could benefit from dose escalation, it likely makes sense to administer additional radioactivity to achieve optimal therapeutic outcomes.

\section{PRIMARY STAKEHOLDERS}

Like any medical service and procedure, dosimetry for RPT needs to meet the requirements and expectations of an array of health-care stakeholders that span the entire billing process. Stakeholder interests should be contextualized in terms of the marginal increase in the cost of care, which overall tends to be dominated by the radiopharmaceutical cost in these procedures. Indeed, Centers for Medicare and Medicaid Services (CMS) reimbursement for ${ }^{177} \mathrm{Lu}-$ DOTATATE or ${ }^{131}$ I-metaiodobenzylguanidine ( $\left.{ }^{131} \mathrm{I}-\mathrm{MIBG}\right)$ often exceeds $\$ 200,000$ for a course of therapy. By comparison, CMS reimbursement for services relating to dosimetry and treatment planning is unlikely to exceed $\$ 10,000-\$ 15,000$ for a course of therapy, depending on the workflow (see "Specific Coding Examples" for details). This represents, at most, a 5\%-7\% increase in the total cost of care. With this as context, stakeholder interests relating to dosimetry are described below.

- The most important group of stakeholders, patients, benefits from improved quality of care. In a given patient, dosimetry-guided RPT has significant potential for toxicity prevention, tumor control improvement, or total avoidance of futile medical intervention.

- Despite the expense of performing dosimetry, medical payers are expected to see a reduction in long-term costs due to avoidance of unnecessary (and typically vastly expensive) cancer therapies in a subset of patients, as well as potential for improved patient outcomes, which further reduces expense liabilities.

- Clinicians stand to benefit from cost-recovery on existing dosimetry practices, reduced liability from adoption of dosimetric guidance (avoiding over-, under-, and futile administration of RPT), and by remaining competitive in offering the highest level of care possible for patients.

- Radiopharmaceutical development and manufacturing entities can benefit from increases in administered activity to patients who stand to benefit most from doing so, and potentially from improved therapeutic windows in late-stage trials (thus reducing the number of patients needed to conduct trials).

- Technical providers of imaging services stand to benefit from increased use of imaging services, in particular existing $\gamma$-camera imaging infrastructure.

- The general public stands to benefit from improved control over the release of radioactive patients, which results in

TABLE 1

List of Common and Emerging Radiopharmaceutical Therapeutics, Their Clinical Indications, and Typical Dose-Limiting Tissues

\begin{tabular}{|c|c|c|}
\hline Radiopharmaceutical & Indication & Dose-limiting tissues \\
\hline${ }^{131}$ I-Nal & Thyroid cancers & Marrow, lungs $(20,21)$ \\
\hline${ }^{90} \mathrm{Y}$-microspheres & $\begin{array}{l}\text { Intrahepatic tumors, including primary and } \\
\text { metastatic disease }\end{array}$ & Liver, lungs, stomach (22) \\
\hline${ }^{177}$ Lu-DOTATATE & Low-grade neuroendocrine tumors & Marrow, kidneys (23) \\
\hline${ }^{131}$ I-MIBG & Paraganglioma, pheochromocytoma & Marrow, kidneys, liver $(24,25)$ \\
\hline${ }^{223} \mathrm{RaCl}_{2}$ & $\begin{array}{l}\text { Metastatic castration-resistant prostate } \\
\text { cancer (mCRPC) }\end{array}$ & Marrow, gastrointestinal (26) \\
\hline${ }^{177}$ Lu-PSMA-617 (investigational) & $\begin{array}{l}\text { Metastatic castration-resistant prostate } \\
\text { cancer (mCRPC) }\end{array}$ & Marrow, salivary glands, kidneys (27) \\
\hline${ }^{177}$ Lu-DOTATOC (investigational) & Low-grade neuroendocrine tumors & Marrow, kidneys (28) \\
\hline${ }^{131}$ I-Iomab-B (investigational) & Acute myeloid leukemia (AML) & Liver (29) \\
\hline
\end{tabular}


approximately 720 person-Sieverts of radiation exposure per year in the United States (34).

\section{DOSIMETRY TECHNIQUES}

\section{General Workflows}

As mentioned in the section "Dosimetry for RPT," multiple data-collection methods are available for dosimetry. Additionally, different radiopharmaceuticals have workflows that are conducive to their typical administration schedule. For example, highspecific-activity ${ }^{131}$ I-MIBG (Azedra; Progenics Pharmaceuticals Inc.) is nominally administered as 2 treatments separated by at least $90 \mathrm{~d}$, whereas ${ }^{177} \mathrm{Lu}$-DOTATATE (Lutathera; Novartis) is administered over 4 therapeutic administrations, each separated by approximately $60 \mathrm{~d}$. Given the goals of using dosimetry to enhance the safety and efficacy of RPT, it is important to have dosimetry results at a time or times in which treatment decisions can be made. In the case of fractionated therapies (e.g., ${ }^{177}$ Lu-DOTATATE [Lutathera])), acquiring dosimetry data after the administration of each therapy can allow for adaptation in subsequent administrations to meet specific treatment planning goals. In the case of high-specific-activity ${ }^{131} \mathrm{I}$-MIBG (Azedra), however, this may or may not be possible, due to potentially reaching or exceeding normal-tissue limits in the first treatment. Likewise, ${ }^{90} \mathrm{Y}$-microsphere therapies are often administered with a single intraarterial infusion, in which case dosimetry and treatment planning are needed before the first therapeutic administration. Therefore, the 2 main dosimetry/treatment planning workflows are as follows:

- Administration of a small amount of the therapeutic, or a predictive surrogate, for purposes of dosimetry and treatment planning before administration of the primary RPT. This workflow is typically used for ${ }^{90} \mathrm{Y}$-microspheres, ${ }^{131} \mathrm{I}-\mathrm{MIBG}$, and ${ }^{131} \mathrm{I}-\mathrm{NaI}$.

- Administration of a full RPT administration, followed by dosimetry for modification of subsequent treatments. This workflow is commonly used for ${ }^{177}$ Lu-DOTATATE and could be used for various agents currently under investigation.

\section{Dosimetric Sampling}

Within a given workflow, a dosimetry schedule should be created based on the needs of a particular RPT and patient. The goal of this schedule should be the accurate determination of dose to relevant tissues (dose-limiting organs or tumors); however, the exact imaging and data-collection sequence will vary with situation. Several specific schedule examples are presented in the section "Specific Coding Examples," however, the following general statements can be made regarding the dosimetry of each agent and tissue type.

${ }^{131} I-N a I$. Thyroid uptake should confirmed and quantified with pretreatment imaging. SPECT/CT and planar $\gamma$-imaging are appropriate for this when using ${ }^{123} \mathrm{I}-\mathrm{NaI}$ or ${ }^{131} \mathrm{I}-\mathrm{NaI}$, and PET/CT is appropriate when using ${ }^{124} \mathrm{I}-\mathrm{NaI}$. Generally, a single imaging time point is adequate for determination of initial tumor uptake fraction; however, quantification of dose to tumor requires anatomic imaging, for example, PET/CT or SPECT/CT, and multiple imaging time points. Quantification of dose to lungs, relevant in cases in which significant lung metastatic disease exists, requires multiple imaging time points and at least 1 anatomic reference scan (e.g., SPECT/CT or PET/CT). Accurate quantification or prediction of marrow dosimetry requires blood sampling at multiple time points and WB planar or SPECT/CT imaging at multiple time points. In summary, a complete and optimal dosimetry workup requires serial blood sampling, serial WB planar imaging, and at least one SPECT/CT that is concordant with one of the planar imaging time points. Some practices have developed population-based biologic clearance models, which may allow for a reduction in the needed data (omission of one or more planar or blood sampling time points); however, these approximations may reduce dosimetric accuracy somewhat (35-37).

${ }^{131}$ I-MIBG and ${ }^{177}$ Lu-DOTATATE. For both agents, marrow and kidney dosimetry are normal organs of interest. Optimal renal dosimetric sampling can be achieved by multiple SPECT/CT imaging time points over the first approximately $7 \mathrm{~d}$ after administration of the therapeutic or a surrogate. Bone marrow dosimetry for these agents can be performed by addition of WB planar imaging and blood sampling at multiple time points. The blood contribution to total marrow dose is less for ${ }^{131} \mathrm{I}-\mathrm{MIBG}$ than for ${ }^{177} \mathrm{Lu}$-DOTATATE, and therefore fewer collections may be needed. Tumor dosimetry, similar to kidney dosimetry, is best performed with serial SPECT/CT imaging. Some investigators have proposed ${ }^{177} \mathrm{Lu}$-DOTATATE imaging time-point reduction strategies for kidneys and tumors $(11,18,19)$; however, these approximations may reduce dosimetric accuracy somewhat (14). Similarly, rather than WB imaging to determine marrow dose from ${ }^{131} \mathrm{I}-\mathrm{MIBG}$, some recommendations include the use of WB counting (rather than imaging) in pediatric patients who would otherwise require general anesthesia for imaging $(38,39)$.

${ }^{223} \mathrm{RaCl}_{2}$. Because of the low administered activity and photon emission abundance, the retention and distribution of ${ }^{223} \mathrm{Ra}$ in a patient is typically assessed by planar imaging only; however, quantitative SPECT/CT has been investigated (40-43).

${ }^{90} Y$-Microspheres. ${ }^{90} \mathrm{Y}$-microspheres are unique among RPTs, due to their nature of maintaining a fixed irradiation geometry after administration. Because of this, only a single imaging time point is needed for dose assessment. For treatment planning purposes, typically ${ }^{99 \mathrm{~m}}$ Tc-macroaggregated albumin ( $\left.{ }^{99 \mathrm{~m}} \mathrm{Tc}-\mathrm{MAA}\right)$ is administered in a way that is consistent with the desired ${ }^{90} \mathrm{Y}$-microsphere administration method (same catheter position in the hepatic arterial tree, same infusion rate). Dose to tumor, liver, and potentially lung and stomach are of interest after this MAA administration. Liver and tumor dosimetric predictions, as well as evaluation for gastric shunting, are made by way of a single SPECT/CT image after administration of MAA. The axial field of view of a single SPECT/CT acquisition is often not adequate for inclusion of the entire lungs, thus lung shunting should be evaluated by collection of an additional SPECT/CT scan, or by conjugate-view planar imaging. In general, lung shunting has been reported to be overestimated by planar imaging, and therefore SPECT/CT may be preferred (44-46). After administration of ${ }^{90} \mathrm{Y}$-microspheres, additional 3D imaging at a single time point (SPECT/CT or PET/CT) is needed to confirm microsphere distribution and associated dosimetry. Some discordance is expected when comparing ${ }^{99 \mathrm{~m}} \mathrm{Tc}-\mathrm{MAA}$ predicted dosimetry and ${ }^{90} \mathrm{Y}$-SPECT/CT estimated dosimetry due to the difference in image quality; however, comparison of these measurements can confirm general treatment distribution and potential eligibility for subsequent ${ }^{90} \mathrm{Y}$-microsphere administrations in the case of progression or undercoverage.

\section{Simplified Dosimetry Methods}

As mentioned above, simplified dosimetry methods for ${ }^{131} \mathrm{I}-\mathrm{NaI}$, ${ }^{177} \mathrm{Lu}$-DOTATATE, and ${ }^{131} \mathrm{I}-\mathrm{MIBG}$ have been proposed $(18,19,35$, 
36,47). These techniques have the potential to reduce the number of imaging sessions or data acquisition requirements, but at the cost of increased dosimetric uncertainty.

In the context of ${ }^{131} \mathrm{I}-\mathrm{NaI}$ dosimetry, work by Hänscheid et al. demonstrated that a single measurement time point 1-2 d after administration maximized the accuracy of marrow dosimetry, with an average residual error of approximately 13\% (35). That said, significant under- and overestimation was observed in some cases, with the estimated/true dose ratio ranging from 0.69 to 1.24 at $24 \mathrm{~h}$. Similar results are described by Jentzen et al., wherein approximately $85 \%$ of patients had residual error of less than $20 \%$, and absolute estimated/true maximum tolerated activity ratios ranged from 0.54 to $1.30(36)$. Work by Jentzen et al. was largely confirmed by Atkins et al. (37). These findings suggest that use of a "simplified" ${ }^{131}$ I dosimetry strategy may require an additional $20 \%-30 \%$ safety margin compared with standard dosimetric sampling.

For ${ }^{177} \mathrm{Lu}$-DOTATATE, data by Sandström et al. indicate that single-time-point renal dosimetry (with an assumption of monoexponential clearance) results in a residual error of less than $20 \%$ in most cases; however, the ratio of estimated to true dose ranges from approximately 0.5 to approximately 1.3 (14). Assumption of biexponential clearance, such as what was originally described by Madsen et al. (48), may improve results somewhat; however, this was not examined by Sandström et al. in their cohort of 777 patients (14). Singletime-point dosimetry has been proposed for other tissues (liver, marrow, tumors) (47); however, further validation is needed.

To date, only 1 publication addresses simplified dosimetry for ${ }^{131}$ I-MIBG (15). The authors concluded that reasonably accurate tumor dosimetry could be achieved using 2 imaging time points; however, further study is needed to evaluate the applicability of single-time-point methods.

\section{Dosimetry Calculations}

Two main methods exist for assessing patient-specific dosimetry, regardless of RPT type: absorbed fraction (e.g., MIRD schema) calculations, and 3D voxelwise dosimetry. These 2 methods are not mutually exclusive, meaning that in a single patient dose to 1 tissue (e.g., bone marrow) may be best assessed by an absorbed fraction calculation, whereas dose to another tissue may be best assessed by a 3D voxelwise dose calculation (e.g., liver). Precise methods and considerations regarding these 2 calculations methods are well described elsewhere (49-52) and thus beyond the scope of this document; however, a general diagram of dosimetry calculation steps, including final treatment plan generation, is shown in Figure 1. As described in the previous section, the exact combination of input data required for dosimetry depends on RPT- and patient-specific factors.
Similarly, the radiation dosimetry and treatment planning workflow will vary depending on specific information required by the physician provider for treatment planning purposes. The primary difference between these 2 dose calculation strategies is that absorbed fraction calculations typically result in mean dose to whole organs, whereas voxelwise calculations can provide a $3 \mathrm{D}$ dose map within the patient anatomy, including isodose lines and dose volume histograms. This distinction is relevant when considering the appropriateness of existing treatment planning current procedural terminology (CPT; a registered trademark of the American Medical Association) codes.

\section{CPT CODE DESCRIPTIONS}

Depending on the specific clinical workflow, personnel effort, documentation, and medical necessity, several existing CPT codes may be applicable to activities relating to radiopharmaceutical dosimetry and treatment planning. A list of existing and potentially pertinent codes and associated relative value units ([RVUs], data obtained from CMS.gov (53)) is provided in Table 2. Detailed

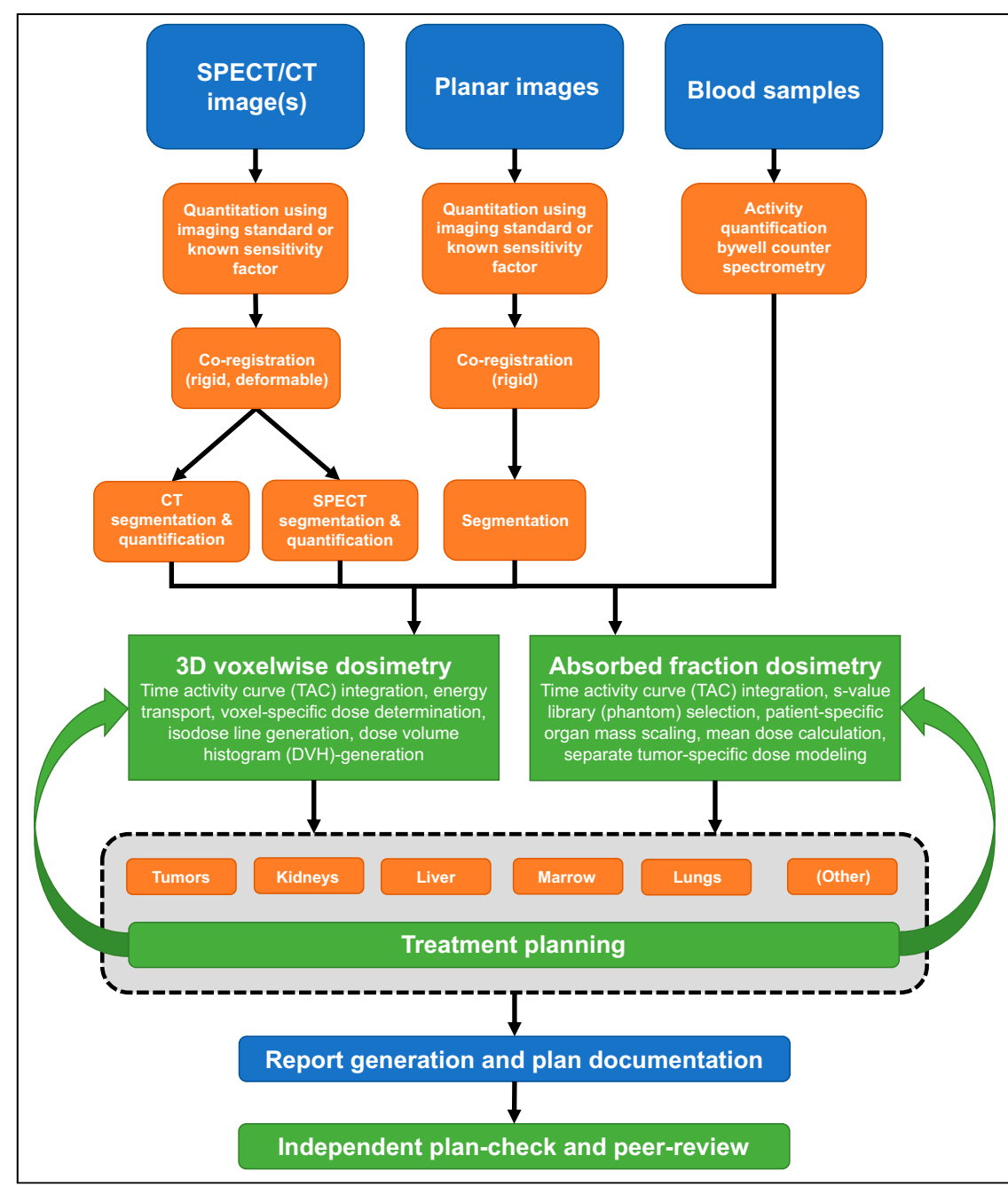

FIGURE 1. Overview of typical RPT dosimetry and treatment planning workflow. Specific input data, dose calculation method, and tissues of interest will depend on the specific radiopharmaceutical and clinical need. Members of the multidisciplinary team responsible for executing the steps of this workflow should be qualified to perform radiopharmaceutical therapy dosimetry, able to interpret dosimetric findings, and able to perform the final treatment plan as documented and reviewed. 
TABLE 2

CPT Codes That May Be Considered Applicable to Radiopharmaceutical Dosimetry and Treatment Planning or Related Activities

\begin{tabular}{|c|c|c|c|c|c|c|c|}
\hline CPT & $\begin{array}{c}\text { Short } \\
\text { description }\end{array}$ & Long description & $\begin{array}{l}\text { Physician } \\
\text { time (min) }\end{array}$ & $\begin{array}{l}\text { Physician } \\
\text { RVU }\end{array}$ & $\begin{array}{l}\text { Physicist/ } \\
\text { technologist } \\
\text { time (min) }\end{array}$ & $\begin{array}{c}\text { Non-facility } \\
\text { RVU }\end{array}$ & $\begin{array}{l}\text { Facility } \\
\text { RVU }\end{array}$ \\
\hline 78800 & $\begin{array}{l}\text { Single area } \\
\text { planar }\end{array}$ & $\begin{array}{l}\text { Radiopharmaceutical localization } \\
\text { of tumor, inflammatory } \\
\text { process or distribution of } \\
\text { radiopharmaceutical agent(s) } \\
\text { (includes vascular flow and } \\
\text { blood-pool imaging, when } \\
\text { performed); planar, single area } \\
\text { (e.g., head, neck, chest, } \\
\text { pelvis), single day imaging }\end{array}$ & 27 & 0.64 & 88 & 7.53 & 2.06 \\
\hline 78801 & $\begin{array}{l}\text { Multiple area } \\
\text { planar }\end{array}$ & $\begin{array}{l}\text { Radiopharmaceutical localization } \\
\text { of tumor, inflammatory } \\
\text { process or distribution of } \\
\text { radiopharmaceutical agent(s) } \\
\text { (includes vascular flow and } \\
\text { blood-pool imaging, when } \\
\text { performed); planar, } 2 \text { or more } \\
\text { areas (e.g., abdomen and } \\
\text { pelvis, head and chest), } 1 \text { or } \\
\text { more days imaging or single } \\
\text { area imaging over } 2 \text { or more } \\
\text { days }\end{array}$ & 30 & 0.73 & 99 & 8.31 & 3.13 \\
\hline 78802 & WB single day & $\begin{array}{l}\text { Radiopharmaceutical localization } \\
\text { of tumor, inflammatory } \\
\text { process or distribution of } \\
\text { radiopharmaceutical agent(s) } \\
\text { (includes vascular flow and } \\
\text { blood-pool imaging, when } \\
\text { performed); planar, WB, single } \\
\text { day imaging }\end{array}$ & 30 & 0.80 & 109 & 9.21 & 4.87 \\
\hline 78804 & $\begin{array}{l}\text { WB } 2 \text { or more } \\
\text { days }\end{array}$ & $\begin{array}{l}\text { Radiopharmaceutical localization } \\
\text { of tumor, inflammatory } \\
\text { process or distribution of } \\
\text { radiopharmaceutical agent(s) } \\
\text { (includes vascular flow and } \\
\text { blood-pool imaging, when } \\
\text { performed); planar, WB, } \\
\text { requiring } 2 \text { or more days } \\
\text { imaging }\end{array}$ & 40 & 1.01 & 216 & 19.42 & - \\
\hline 78803 & $\begin{array}{l}\text { SPECT single } \\
\text { area/single } \\
\text { day }\end{array}$ & $\begin{array}{l}\text { Radiopharmaceutical localization } \\
\text { of tumor, inflammatory } \\
\text { process or distribution of } \\
\text { radiopharmaceutical agent(s) } \\
\text { (includes vascular flow and } \\
\text { blood-pool imaging, when } \\
\text { performed); tomographic } \\
\text { (SPECT), single area (e.g., } \\
\text { head, neck, chest, pelvis), } \\
\text { single day imaging }\end{array}$ & 42 & 1.09 & 130 & 11.38 & - \\
\hline 78830 & $\begin{array}{l}\text { SPECT/CT } \\
\text { single area/ } \\
\text { single day }\end{array}$ & $\begin{array}{l}\text { Radiopharmaceutical localization } \\
\text { of tumor, inflammatory } \\
\text { process or distribution of } \\
\text { radiopharmaceutical agent(s) } \\
\text { (includes vascular flow and } \\
\text { blood-pool imaging, when } \\
\text { performed); tomographic } \\
\text { (SPECT) with concurrently } \\
\text { acquired CT transmission } \\
\text { scan for anatomic review, } \\
\text { localization and } \\
\text { determination/detection of } \\
\text { pathology, single area (e.g., } \\
\text { head, neck, chest, pelvis), } \\
\text { single day imaging }\end{array}$ & 45 & 1.49 & 141 & 14.46 & - \\
\hline
\end{tabular}


TABLE 2

CPT Codes That May Be Considered Applicable to Radiopharmaceutical Dosimetry and Treatment Planning or Related Activities (cont.)

\begin{tabular}{|c|c|c|c|c|c|c|c|}
\hline CPT & $\begin{array}{c}\text { Short } \\
\text { description }\end{array}$ & Long description & $\begin{array}{l}\text { Physician } \\
\text { time (min) }\end{array}$ & $\begin{array}{l}\text { Physician } \\
\text { RVU }\end{array}$ & $\begin{array}{l}\text { Physicist/ } \\
\text { technologist } \\
\text { time (min) }\end{array}$ & $\begin{array}{l}\text { Non-facility } \\
\text { RVU }\end{array}$ & $\begin{array}{l}\text { Facility } \\
\text { RVU }\end{array}$ \\
\hline 78831 & $\begin{array}{l}\text { SPECT } \\
\text { minimum of } 2 \\
\text { areas in } 1 \mathrm{~d} \\
\text { or single are } \\
\text { over } 2 \text { or } \\
\text { more days }\end{array}$ & $\begin{array}{l}\text { Radiopharmaceutical localization } \\
\text { of tumor, inflammatory } \\
\text { process or distribution of } \\
\text { radiopharmaceutical agent(s) } \\
\text { (includes vascular flow and } \\
\text { blood-pool imaging, when } \\
\text { performed); tomographic } \\
\text { (SPECT), minimum } 2 \text { areas } \\
\text { (e.g., pelvis and knees, } \\
\text { abdomen and pelvis), single } \\
\text { day imaging, or single area } \\
\text { imaging over } 2 \text { or more days }\end{array}$ & 55 & 1.82 & 224 & 20.87 & - \\
\hline 78832 & $\begin{array}{l}\text { SPECT/CT } \\
\text { minimum of } 2 \\
\text { areas in } 1 \mathrm{~d} \\
\text { or single are } \\
\text { over } 2 \text { or } \\
\text { more days }\end{array}$ & $\begin{array}{l}\text { Radiopharmaceutical localization } \\
\text { of tumor, inflammatory } \\
\text { process or distribution of } \\
\text { radiopharmaceutical agent(s) } \\
\text { (includes vascular flow and } \\
\text { blood pool imaging, when } \\
\text { performed); tomographic } \\
\text { (SPECT) with concurrently } \\
\text { acquired CT transmission } \\
\text { scan for anatomic review, } \\
\text { localization and } \\
\text { determination/detection of } \\
\text { pathology, minimum } 2 \text { areas } \\
\text { (e.g., pelvis and knees, } \\
\text { abdomen and pelvis), single } \\
\text { day imaging, or single area } \\
\text { imaging over } 2 \text { or more days }\end{array}$ & 60 & 2.12 & 264 & 27.19 & - \\
\hline 78835 & $\begin{array}{l}\text { Quantification } \\
\text { for SPECT/ } \\
\text { CT (use with } \\
78830 \text { or } \\
78832 \text { ) } \\
{ }^{*} \text { report } \\
\text { multiple units }\end{array}$ & $\begin{array}{l}\text { Radiopharmaceutical } \\
\text { quantification measurement(s) } \\
\text { single area (list separately in } \\
\text { addition to code for primary } \\
\text { procedure) }\end{array}$ & 17 & 0.47 & 23 & 3.00 & - \\
\hline 78814 & Limited PET/CT & $\begin{array}{l}\text { PET with concurrently acquired } \\
\text { CT for attenuation correction } \\
\text { and anatomic localization } \\
\text { imaging; limited area (e.g., } \\
\text { chest, head/neck) }\end{array}$ & 60 & 2.20 & Carrier-priced & 0.00 & - \\
\hline 78580 & - & $\begin{array}{l}\text { Pulmonary perfusion imaging } \\
\text { (e.g., particulate) }\end{array}$ & 20 & 0.74 & 93 & 6.96 & \\
\hline 77300 & $\begin{array}{l}\text { Basic radiation } \\
\text { dosimetry } \\
\text { calculation }\end{array}$ & $\begin{array}{l}\text { Basic radiation dosimetry } \\
\text { calculation, central axis depth } \\
\text { dose calculation, time-dose } \\
\text { factor, nominal standard } \\
\text { dose, gap calculation, off axis } \\
\text { factor, tissue inhomogeneity } \\
\text { factors, calculation of } \\
\text { nonionizing radiation surface } \\
\text { and depth dose, as required } \\
\text { during course of treatment, } \\
\text { only when prescribed by the } \\
\text { treating physician }\end{array}$ & 15 & 0.62 & 14 & 1.93 & - \\
\hline $77370^{3}$ & - & $\begin{array}{l}\text { Special medical radiation } \\
\text { physics consultation }\end{array}$ & 0 & 0.00 & 65 & 3.75 & - \\
\hline 77261 & - & $\begin{array}{l}\text { Treatment planning: (simple, } \\
\text { intermediate, complex) }\end{array}$ & 36 & 1.30 & - & 2.06 & 2.06 \\
\hline 77262 & & & 54 & 2.00 & - & 3.13 & 3.13 \\
\hline 77263 & & & 82 & 3.14 & - & 4.87 & $\begin{array}{c}4.87 \\
\text { ontinued) }\end{array}$ \\
\hline
\end{tabular}


TABLE 2

CPT Codes That May Be Considered Applicable to Radiopharmaceutical Dosimetry and Treatment Planning or Related Activities (cont.)

\begin{tabular}{|c|c|c|c|c|c|c|c|}
\hline СРТ & $\begin{array}{c}\text { Short } \\
\text { description }\end{array}$ & Long description & $\begin{array}{l}\text { Physician } \\
\text { time (min) }\end{array}$ & $\begin{array}{c}\text { Physician } \\
\text { RVU }\end{array}$ & $\begin{array}{l}\text { Physicist/ } \\
\text { technologist } \\
\text { time (min) }\end{array}$ & $\begin{array}{c}\text { Non-facility } \\
\text { RVU }\end{array}$ & $\begin{array}{c}\text { Facility } \\
\text { RVU }\end{array}$ \\
\hline 77295 & - & $\begin{array}{l}\text { 3-dimensional radiation } \\
\text { treatment plan, including } \\
\text { dose-volume histograms }\end{array}$ & 112 & 4.29 & 165 & 14.07 & - \\
\hline
\end{tabular}

Stochastic effects, such as secondary hematologic malignancies, have also been shown to result from radiation exposure and chemotherapy. Current models suggest that these effects are not associated with a dose threshold, but rather the effect risk is thought to increase with increasing cumulative treatment. Rather than individualized dosimetry for toxicity avoidance, stochastic effects are better informed by population-level dosimetry data for risk modeling.

examples of these codes are included in the section "Specific Coding Examples"; however, the codes can generally be divided into those for $\gamma$-imaging $(78800,78801,78802,78803,78804,78830$, $78831,78832,78580)$, PET imaging (78814), dosimetry and treatment planning $(77300,77261,77262,77263,77295)$, and ancillary services $(77370,78835)$. Notable exclusions from the table below include more general PET imaging codes (i.e., 78811, 78812, $78813,78815,78816)$, which might be applicable in the case where PET or PET/CT is used for pretreatment dosimetry. Codes relating to brachytherapy dosimetry and treatment planning (i.e., $77316,77317,77318)$ may also be considered applicable in some situations.

\section{SPECIFIC CODING EXAMPLES}

Below is a series of clinical workflows that may be encountered, including reimbursement coding that is relevant to imaging, dosimetry, and treatment planning. We have intentionally omitted descriptions and codes related to radiopharmaceuticals, radiopharmaceutical administrations, patient consultation, and follow-up. Coding for these related activities are left for other documents.

\section{Example 1. ${ }^{90} \mathrm{Y}$-Radioembolization (with Pretreatment} ${ }^{99 m}$ Tc-MAA Mapping)

A patient was determined to be a candidate for ${ }^{90} \mathrm{Y}$ radioembolization. Dosimetric planning for treatment began by preparation of a calibrated quantity of ${ }^{99 \mathrm{~m}}$ Tc-MAA. An interventional radiologist localized a catheter to a satisfactory location within the arterial supply of a liver for infusion of the ${ }^{99 \mathrm{~m}} \mathrm{Tc}-\mathrm{MAA}$. Catheter tip placement was optimized based on tumor location and the perfused volume indicated by iodine-enhanced digital subtraction fluoroscopy or cone beam CT imaging in the interventional suite. After infusion of the ${ }^{99 \mathrm{~m}} \mathrm{Tc}-\mathrm{MAA}$, the patient was relocated to a SPECT/CT scanner for imaging. Acquired were SPECT/CT images centered on the liver (78830) and conjugate planar images covering the extent of lungs and liver (bundled with 78830).

After acquisition, it was confirmed by the authorized user and treating physician that the perfused volume within the liver was appropriate for therapy. A decision was made regarding sphere type (glass vs. resin) based on desired sphere specific activity and specific gravity.

Lung shunt fraction (LSF) was calculated by manually drawing regions of interest (ROIs) on conjugate planar views - lungs, liver, and corresponding background ROIs. Counts quantified in each region were corrected for background, a geometric mean was calculated for the lungs and liver separately, and the fraction of total activity in the lungs was calculated (78835 for each ROI). On the basis of institutional policy, this LSF was considered sufficiently low that a more accurate $3 \mathrm{D}$ evaluation was not needed. This LSF was transcribed in the patient medical record.

Dosimetry proceeded by use of $510(\mathrm{k})$-cleared medical device software for 3D microsphere dosimetry. A physicist, physician, or another qualified individual segmented the whole liver, the perfused portion of the liver, the tumor, and the tumor plus a planning margin to account for breathing motion and potential microinvasion. A 3D dose plan normalized to a nominal administered activity was reviewed by the authorized user, and it was determined that an administered activity of $3.52 \mathrm{GBq}$ was appropriate to maximize tumor dose, without exceeding dose limits to normal liver parenchyma. Three-dimensional dosimetry statistics for this final treatment plan were generated, and a treatment plan report was generated and signed by the physicist and authorized user (77295). An independent qualified individual reviewed this plan for appropriateness and accuracy, including performing a simplified dose calculation via the partition model. This secondary dose verification was documented in the medical record (77300).

Approximately $2 \mathrm{wk}$ after the initial mapping procedure, the patient returned for treatment. The interventional radiologist placed the catheter tip at the same location within the liver arterial vasculature, and ${ }^{90} \mathrm{Y}$-microspheres were infused according to manufacturer-recommended methods. Because stasis was reached during administration, only $3.24 \mathrm{GBq}$ were administered. The patient was transferred for posttreatment Bremsstrahlung SPECT/ CT imaging, whereby a single SPECT/CT view, centered on the liver (78830), and a conjugate planar image including the extent of lungs and liver (bundled with 78830) were acquired. On the basis of these images, dosimetry was performed to assess the delivered dose (77300).

The following is a summary of the procedure and corresponding CPT codes:

- MAA mapping SPECT/CT and planar, 78830;

- Lung shunt quantification, 78835 (2 units);

- 3D radiation treatment planning, 77295 ;

- Plan check/simple dosimetry, 77300;

- ${ }^{90} \mathrm{Y}$ SPECT/CT and planar, 78830; and

- Treatment verification (simple dosimetry), 77300.

Comments. If posttreatment ${ }^{90} \mathrm{Y}$ PET/CT imaging covering the liver and lungs is performed rather than posttreatment SPECT/CT 
and planar, 78814 (limited area PET/CT) would take the place of the posttreatment imaging code 78830. If pretreatment MAA mapping is not performed, the pretreatment imaging, LSF assessment, treatment planning codes, and treatment plan verification codes $(78830,78835,77295,77300)$ would not be applicable. If treatment planning is performed using methods other than a full-3D voxelwise calculation (e.g., partition method or whole liver mean dose determination), simple, intermediate, or complex treatment planning codes $(77261,77262$, or 77263$)$ should be used in place of 77295 . If SPECT/CT is not acquired after ${ }^{99 \mathrm{~m}}$ Tc-MAA administration, 78800 or 78801 for the planar imaging would be billed in lieu of 78830. Assuming lung shunt quantification is performed from the planar images, 78580 could be used in lieu of 78835 units. Additionally, 77295 would no longer be applicable (see the earlier text).

\section{Example 2. ${ }^{177}$ Lu-DOTATATE (with Tumor, Marrow, and Kidney Dosimetry)}

After clinical evaluation and results from diagnostic ${ }^{68} \mathrm{Ga}$ DOTATOC PET/CT imaging, a patient was deemed to be eligible for treatment with ${ }^{177} \mathrm{Lu}$-DOTATATE RPT. No pretreatment dosimetry was performed; however, eligibility for subsequent ${ }^{177} \mathrm{Lu}$-DOTATATE administrations (every $8 \mathrm{wk}$ ) would be determined on the basis of prior and cumulative radiation doses from treatment.

The patient presented for the first therapeutic administration. The patient had a peripheral intravenous catheter placed, and an infusion of nephroprotective amino acids was started. After approximately 30 min had elapsed ( $200 \mathrm{cc}$ of fluid infused), it was confirmed by a nuclear medicine technologist that no signs of extravasation were present. The RPT (7.4 GBq of ${ }^{177}$ Lu-DOTATATE) was administered through the same intravenous catheter by standard institutional practice, and the amino acid infusion proceeded until completion, approximately $4 \mathrm{~h}$ after the start of infusion.

After completion of RPT administration, a blood sample was collected for dosimetric purposes. The patient was monitored and released. In the following days, at $24 \mathrm{~h}$ after injection, $72 \mathrm{~h}$ after injection, and $120 \mathrm{~h}$ after injection, the patient returned for dosimetric sampling. Each dosimetric sampling consisted of blood collection, WB planar imaging (78804), and abdominal SPECT/CT (78832). On the basis of the 24-h SPECT/CT acquisition, it was determined that extravasation of ${ }^{177} \mathrm{Lu}$ had not occurred.

Dosimetry proceeded by use of $510(\mathrm{k})$-cleared medical device software for generalized 3D RPT dosimetry. A physicist, physician, or another qualified individual segmented organs of interest (whole liver, spleen, kidneys) as well as the 3 largest tumor lesions. Marrow dosimetry was performed by absorbed-fraction (MIRD) methods based on WB, blood, and normal organ timeintegrated-activity quantification. A 3D dose plan normalized to a nominal administered activity was reviewed by the authorized user, and it was determined that a cumulative administered activity of $25.5 \mathrm{GBq}$ was appropriate to maximize tumor dose, without exceeding dose limits to normal tissues (kidneys, liver, marrows) and within the limits of radiopharmaceutical availability. Three-dimensional dosimetry statistics for this final treatment plan were generated, and a treatment plan report was generated and signed by the physicist and authorized user (77295). An independent qualified individual reviewed this plan for appropriateness and accuracy, including performing a simple dose calculation via established absorbed-fraction (MIRD) methods. This secondary dose verification was documented in the medical record (77300).

After $8 \mathrm{wk}$, the patient returned for the second (Tx 2). On the basis of the target cumulative administered activity of $25.5 \mathrm{GBq}$, the patient was deemed eligible for an additional full administration of $7.4 \mathrm{GBq}$. The treatment was administered, and the patient underwent the same dosimetric sampling regimen as described above. Posttreatment dosimetry was performed to evaluate for deviation from expected tumor and normal organ doses. On the basis of compliance with the original treatment plan, treatment 3 (Tx 3) proceeded in the same manner with a $7.4 \mathrm{GBq}$ administration and posttreatment dosimetry. The final treatment (Tx 4) was delivered in compliance with the initial treatment plan, with an administered activity of $3.3 \mathrm{GBq}$ (cumulative $25.5 \mathrm{GBq}$ ). After the terminal treatment, dosimetric sampling was repeated. On the basis of these data, dosimetry was performed and combined with results from all 4 treatments (77300). A final patient-specific dose report was generated and documented in the medical record.

The following is a summary of the procedure and corresponding CPT codes:

- Tx $1{ }^{177} \mathrm{Lu}$ WB planar imaging (3 d), 78804;

- Tx $1{ }^{177} \mathrm{Lu}$ abdominal SPECT/CT (3 d), 78832;

- 3D radiation treatment planning, 77295 ;

- Plan check/simple dosimetry, 77300;

- Tx $2{ }^{177} \mathrm{Lu}$ WB planar imaging (3 d), 78804;

- Tx $2{ }^{177} \mathrm{Lu}$ abdominal SPECT/CT (3 d), 78832;

- Dosimetry assessment, 77300;

- Tx $3{ }^{177} \mathrm{Lu}$ WB planar imaging (3 d), 78804;

- Tx $3{ }^{177} \mathrm{Lu}$ abdominal SPECT/CT (3 d), 78832;

- Dosimetry assessment, 77300;

- Tx $4{ }^{177} \mathrm{Lu}$ WB planar imaging (3 d), 78804;

- Tx $4{ }^{177} \mathrm{Lu}$ abdominal SPECT/CT (3 d), 78832; and

- Dosimetry assessment, 77300

Comments. If tumors are not included within the abdominal SPECT/CT field of view, and tumor dosimetry is needed, additional SPECT fields of view would be required. This would not change the coding unless only a single posttreatment SPECT/CT were planned, in which case 78832 would be submitted in lieu of 78830. If marrow dosimetry is not performed, WB planar imaging (78804) and blood sampling should be omitted unless otherwise deemed medically necessary.

\section{Example 3. ${ }^{177}$ Lu-DOTATATE (Dialysis Patient, Marrow Dosimetry)}

After clinical evaluation and results from diagnostic ${ }^{68} \mathrm{Ga}-$ DOTATOC PET/CT imaging, a patient was deemed to be eligible for treatment with ${ }^{177} \mathrm{Lu}$-DOTATATE RPT. In addition to having advanced neuroendocrine tumors, this patient had poor kidney function due to obstruction and was therefore receiving hemodialysis $3 \mathrm{~d}$ per week. Because of the compromised kidney function, blood clearance of any therapeutic radiopharmaceutical was expected to be significantly inhibited compared with the typical patient presentation. For this reason, the decision was made to initially administer $3.7 \mathrm{GBq}$ of ${ }^{177} \mathrm{Lu}$-DOTATATE (rather than the standard $7.4 \mathrm{GBq}$ ), followed by bone marrow dosimetry to develop a treatment plan for subsequent administrations. For this patient, treatment was deemed to be palliative, and therefore kidney, liver, and tumor dosimetry were considered to be secondarily important to the most likely normal-tissue toxicity (bone marrow). 
RPT was administered (methods consistent with what was described in Example 6.2), and the following dosimetric sampling was performed:

- Blood sampling at $4 \mathrm{~h}$ after administration (end of AA infusion);

- Hemodialysis performed from 4.5 to $7 \mathrm{~h}$ after administration;

- Blood sampling at $7.5 \mathrm{~h}$ after administration;

- Blood sampling and WB conjugate planar imaging at $24 \mathrm{~h}$ after administration;

- Blood sampling and WB conjugate planar imaging at $46 \mathrm{~h}$ after administration;

- Hemodialysis performed from 47 to $49 \mathrm{~h}$ after administration;

- Blood sampling performed at $49 \mathrm{~h}$ after administration; and

- Blood sampling and WB conjugate planar imaging at $96 \mathrm{~h}$ after administration.

After completion of dosimetric sampling, a special medical physics consult (77370) was ordered by the treating physician. The consult request was made to evaluate the effect of hemodialysis and blood retention of ${ }^{177}$ Lu-DOTATATE and associated marrow dosimetric effects. Dosimetry calculations were performed by a qualified medical physicist in addition to evaluating the impact of dialysis, and a consultation report was generated and documented. It was determined that minimal blood clearance occurred between dialysis sessions. Absorbed-fraction (MIRD)-based marrow dosimetry indicated significant elevation of population average dose values (more than 4 times the approved label average value). On the basis of this analysis performed by the qualified medical physicist, it was determined that addition of a hemodialysis session at $24 \mathrm{~h}$ after administration would be beneficial for marrow dosimetry due to increased peptide removal after the initial tumor uptake phase. A treatment plan was developed (77262) that included this modification while targeting a total administered activity of $14.8 \mathrm{GBq}$, with the remaining activity $(11.1 \mathrm{GBq})$ to be split between treatments 2 and 3. An independent qualified individual reviewed this plan for appropriateness and accuracy, including performing a simple dose calculation via established absorbed-fraction (MIRD) methods. This secondary dose verification was documented in the medical record (77300).

Treatments 2 and 3 were completed according to the treatment plan, with postadministration dosimetry performed as described above. Bone marrow dosimetry (77300) was performed after each treatment, with dose reports (fraction and cumulative) being documented in the patient medical record.

The following is a summary of the procedure and corresponding CPT codes:

- Tx $1{ }^{177} \mathrm{Lu}$ WB planar imaging (3 d), 78804;

- Med physics special consult: dialysis pharmacokinetics, 77370;

- Treatment planning (intermediate), 77262;

- Tx 1 marrow dosimetry, 77262;

- Tx 2 177Lu WB planar imaging (3 d), 78804;

- Tx 2 marrow dosimetry, 77300;

- Tx 3 177Lu WB planar imaging (3 d), 78804; and

- Tx 3 marrow dosimetry, 77300 .

Comments. The choice of 77262 (intermediate) rather than 77261 (simple) or 77263 (complex) in this example is based on the time-sensitive nature of radiation dose delivery, including appropriate timing of dialysis; however, this treatment plan did not consider many specific treatment areas or organs at risk, and therefore 77263 would likely not be appropriate. A reduction in administered activity in this example led to elimination of 1 treatment administration compared with standard administration workflows - this led to substantial and immediate payer cost savings, and reduced risk of severe toxicity experienced by the patient.

\section{Example 4. ${ }^{131}$ I-MIBG (with Tumor, Marrow, and Kidney Dosimetry)}

After clinical evaluation and results from diagnostic ${ }^{123} \mathrm{I}-\mathrm{MIBG}$ SPECT/CT imaging, a patient was deemed to be eligible for treatment with ${ }^{131}$ I-MIBG RPT. Per the Food and Drug Administrationapproved label for this RPT, pretreatment dosimetry was performed using a small quantity of the therapeutic radiopharmaceutical.

Radiopharmaceutical was administered (185 MBq) in a manner consistent with manufacturer recommendations and institutional policy. After administration, dosimetric sampling was collected. Dosimetric sampling consisted of blood sample collection at 4, 24, 48, and $96 \mathrm{~h}$ after administration and imaging (WB conjugate planar + SPECT/CT of the abdomen) at 24, 48, and $96 \mathrm{~h}$ after administration.

Dosimetry proceeded by use of $510(\mathrm{k})$-cleared medical device software for generalized 3D RPT dosimetry. A physicist, physician, or another qualified individual segmented organs of interest (whole liver, spleen, kidneys) as well as the 3 largest tumor lesions. Marrow dosimetry was performed by absorbed-fraction (MIRD) methods based on WB, blood, and normal organ timeintegrated-activity quantification. A 3D dose plan normalized to a nominal administered activity was reviewed by the authorized user, and it was determined that a cumulative administered activity of $26 \mathrm{GBq}$ was appropriate to maximize tumor dose, without exceeding dose limits to normal tissues (in this case bone marrow). ${ }^{131} \mathrm{I}-\mathrm{MIBG}$ is typically administered over 2 treatments, and therefore a plan of administering $13 \mathrm{GBq}$ in each treatment, separated by at least $90 \mathrm{~d}$. Three-dimensional dosimetry statistics for this final treatment plan were generated, and a treatment plan report was generated and signed by the physicist and authorized user (77295). An independent qualified individual reviewed this plan for appropriateness and accuracy, including performing a simple dose calculation via established absorbed-fraction (MIRD) methods. This secondary dose verification was documented in the medical record (77300).

The patient returned for initial treatment. In accordance with the treatment plan, $13 \mathrm{GBq}$ of ${ }^{131} \mathrm{I}-\mathrm{MIBG}$ was administered. The patient underwent posttreatment dosimetric sampling with the same blood collection and imaging time points as described above. Dosimetry was performed (77300) over the $96 \mathrm{~h}$ after administration, and a posttreatment dose report was documented and reviewed by the treating physician (77300). On the basis of exposure rate measurements, the patient was retained with "in-patient" status until the end of day 2, at which time the patient met Nuclear Regulatory Commission and state release criteria. Before and after release, the patient was monitored for treatment-related adverse events.

After $90 \mathrm{~d}$ had elapsed, the patient returned for an additional treatment of $13 \mathrm{GBq}$ ( $26 \mathrm{GBq}$ cumulative) in accordance with the treatment plan. Dosimetry was again performed (77300), with a final cumulative dose report being generated, documented, and reviewed by the treating physician.

The following is a summary of the procedure and corresponding CPT codes: 
- Pre-Tx 131I WB planar imaging (3 d), 78804;

- Pre-Tx 131I abdominal SPECT/CT (3 d), 78832;

- 3D radiation treatment planning, 77295 ;

- Plan check/simple dosimetry, 77300;

- Tx 1 131I WB planar imaging (3 d), 78804;

- Tx 1 131I abdominal SPECT/CT (3 d), 78832;

- Dosimetry assessment, 77300;

- Tx 2 131I WB planar imaging (3 d), 78804;

- Tx 2 131I abdominal SPECT/CT (3 d), 78832; and

- Dosimetry assessment, 77300

Comments. Many patients who can benefit form ${ }^{131} \mathrm{I}-\mathrm{MIBG}$ therapy are quite young (below the age of 4 ), and therefore require general anesthesia for dosimetric imaging. In these cases the treating physician, in collaboration with the multidisciplinary team, may choose to forgo dosimetry after Tx 1 and Tx 2, or develop a nonstandard pretreatment dosimetry workflow in consultation with a qualified medical physicist (77370). An example of a modified dosimetric sampling would be standard blood collections; WB planar imaging at $24 \mathrm{~h}$; and WB counting (nonanesthetized) at 4, 24, 48 , and $96 \mathrm{~h}$ after administration. In general, these modifications preclude tumor dosimetry; however, dose to the primary limiting organ (bone marrow) can be assessed with reduced precision. Additional details regarding abbreviated dosimetry methods can be found in the EANM procedure guidelines for ${ }^{131} \mathrm{I}-\mathrm{MIBG}$ therapy (39).

\section{CURRENT DEFICIENCIES AND FUTURE NEEDS}

Although the coding strategies described herein are appropriate to meet the immediate need for baseline support of dosimetry and treatment planning for RPT, the existing CPT code set does not contain a sufficient spectrum of codes to describe the current and anticipated process of care for RPT procedures. Some services fit within the scope of existing codes; however, many services remain unsupported or undersupported by existing codes. New and dedicated codes for theranostics should be developed, with collaboration between relevant stakeholders (Society of Nuclear Medicine and Molecular Imaging [SNMMI], American Society for Radiation Oncology [ASTRO], American College of Radiology [ACR], Society of Interventional Radiology [SIR], American Association of Physicists in Medicine [AAPM], and others). What follows are several notable deficiencies among the current coding structure; however. this list is neither intended to be comprehensive nor authoritative.

\section{Partition and Volume-Based ${ }^{90} \mathrm{Y}$-Microsphere Treatment Planning}

Although 77295 may be appropriate when the clinical case rises to a level of complexity requiring generation and review of $3 \mathrm{D}$ isodose volumes relative to normal tissue and tumor targets, a common method of calculation in somewhat simpler cases (e.g., single lesion, well-defined uptake, limited volume of perfusion) involves an approximation of uniform activity distribution in the target tumor and normal liver. Under this approximation, one must determine the volume of treated liver + tumor, the total liver volume, the tumor-to-liver concentration ratio, and the fraction of activity shunting to lungs and other normal tissues. On the basis of these data, calculations can be performed to provide a range of potential treatment plans, from which the authorized user can select the most appropriate. The effort for these activities may exceed what is included in 77261-77263, and thus new codes may need to be developed based on plan complexity.

\section{WB Counting}

In some cases, particularly pediatric patients, it may be more appropriate to use serial WB counting in lieu of serial WB planar imaging for the purposes of bone marrow dose assessment. WB counting may involve use of a scintillation spectrometer (i.e., shielded NaI thyroid uptake probe) or use of an ion chamber survey meter. The WB counting procedures, which can allow for data acquisition without general anesthesia in pediatric patients, is not currently supported by any existing code.

\section{More Than 2 SPECT/CT or WB Planar Scans}

As indicated by examples provided in the section "Specific Coding Examples" in this paper, it is sometimes necessary to obtain more than 2 imaging fields of view (areas) or imaging time points to adequately characterize the spatial and temporal distribution of radiopharmaceutical in organs and tumors of interest. 78804 and 78832 provide reimbursement for only 2 WB planar images and SPECT/CT areas or imaging timepoints, respectively. Revision of these code, or creation of a modifier to account for additional timepoints, should be undertaken.

\section{RPT "Simulation"}

Although not commonly performed (with the exception of ${ }^{131}$ I-MIBG), administration of a small quantity of the therapeutic radiopharmaceutical or an appropriate surrogate followed by dosimetric sampling may emerge as a useful technique for treatment planning. This process may or may not include supportive compounds, such as infusion of renal-protective amino acids, as these compounds are known to alter the pharmacokinetics of the therapeutic radiopharmaceutical. Dedicated codes for this workflow and associated radiopharmaceutical costs may be needed.

\section{Blood Collection and Counting}

Analysis of biologic samples is needed for determination of bone marrow dose in most cases. Analysis may include wholeblood spectroscopic counting, plasma spectroscopic counting, and determination of the patient's hematocrit. These procedures, typically performed by a nuclear medicine technologist, appear unsupported by the current code set.

\section{Sequential PET/CT for RPT}

Interest is growing in the use of positron-emitting surrogates for RPTs, such as ${ }^{64} \mathrm{Cu}$-DOTATATE as a surrogate for ${ }^{177} \mathrm{Lu}$-DOTATATE. Currently there are no codes for multiple-time-point PET/ CT imaging, such as what is available for SPECT/CT.

\section{Consensus Regarding "Simplified" Dosimetry}

As discussed in the section "Primary Stakeholders," there are some emerging data suggesting that adequate dosimetry can be performed from a limited number of imaging time points based on population pharmacokinetic data. These emerging techniques should be examined by experts in the field, to develop consensus recommendations regarding which clinical scenarios are wellsuited to simplification or time-point reduction.

\section{DISCLAIMER}

The opinions provided in this paper are those of members of the SNMMI Dosimetry Task Force based on their coding experience. Always check with your local insurance carriers, as policies vary 
by region. The billing strategies described in this paper are unlikely to be accepted universally, and so the final decision for coding for any procedure must be made by the physician, considering regulations of insurance carriers and any local, state or federal laws that apply to the physician's practice. Neither SNMMI nor any of its officers, directors, agents, employees, committee members, or other representatives shall have any liability for any claim, whether founded or unfounded, of any kind whatsoever, including but not limited to any claim for costs and legal fees, arising from the use of these opinions.

\section{DISCLOSURE}

Denise A.M. Merlino is a consultant of the SNMMI. No other potential conflict of interest relevant to this article was reported.

\section{ACKNOWLEDGMENTS}

We are grateful for input from ASTRO and ACR representatives, as well as support and input from the SNMMI Dosimetry Task Force.

\section{KEY POINTS}

QUESTION: Is it possible to obtain reimbursement for radiopharmaceutical dosimetry?

PERTINENT FINDINGS: Existing reimbursement codes are applicable to several RPT dosimetry procedures, provided the code description matches the activity being performed. Some activities are not well supported by existing codes, and efforts should be made to address this need.

IMPLICATIONS FOR PATIENT CARE: Cost recovery for radiopharmaceutical dosimetry will allow for widespread adoption, thus leading to improved clinical management of patients.

\section{REFERENCES}

1. Becquerel H, Curie P. Action physiologique des rayons du radium. Compt Rend Acad Sci. 1901;132:1289-1291.

2. Daniel J. The x-rays. Science. 1896;3:562-563.

3. Strigari L, Konijnenberg M, Chiesa C, et al. The evidence base for the use of internal dosimetry in the clinical practice of molecular radiotherapy. Eur J Nucl Med Mol Imaging. 2014;41:1976-1988.

4. Wessels BW, Konijnenberg MW, Dale RG, et al. MIRD pamphlet no. 20: the effect of model assumptions on kidney dosimetry and response: implications for radionuclide therapy. J Nucl Med. 2008;49:1884-1899.

5. Chiesa C, Mira M, Bhoori S, et al. Radioembolization of hepatocarcinoma with 90 $\mathrm{Y}$ glass microspheres: treatment optimization using the dose-toxicity relationship. Eur J Nucl Med Mol Imaging. 2020;47:3018-3032.

6. Violet J, Jackson P, Ferdinandus J, et al. Dosimetry of ${ }^{177}$ Lu-PSMA-617 in metastatic castration-resistant prostate cancer: correlations between pretherapeutic imaging and whole-body tumor dosimetry with treatment outcomes. J Nucl Med. 2019;60:517-523.

7. Garin E, Tselikas L, Guiu B, et al. Personalised versus standard dosimetry approach of selective internal radiation therapy in patients with locally advanced hepatocellular carcinoma (DOSISPHERE-01): a randomised, multicentre, openlabel phase 2 trial. Lancet Gastroenterol Hepatol. 2021;6:17-29.

8. Ilan E, Sandström M, Wassberg C, et al. Dose response of pancreatic neuroendocrine tumors treated with peptide receptor radionuclide therapy using ${ }^{177} \mathrm{Lu}-\mathrm{DO}-$ TATATE. J Nucl Med. 2015;56:177-182.

9. Pauwels S, Barone R, Walrand S, Borson-Chazot F. Practical dosimetry of peptide receptor radionuclide therapy with $^{\circ}$ sup $90^{\circ}$ Y-labeled somatostatin analogs. J Nucl Med. 2005;46:92S.

10. Chansanti O, Jahangiri Y, Matsui Y, et al. Tumor dose response in Yttrium-90 resin microsphere embolization for neuroendocrine liver metastases: a tumor- specific analysis with dose estimation using SPECT-CT. $J$ Vasc Interv Radiol. 2017;28:1528-1535.

11. Hou X, Brosch J, Uribe C, et al. Feasibility of single-time-point dosimetry for radiopharmaceutical therapies. $J$ Nucl Med. 2021;62:1006-1011.

12. Menda Y, Madsen MT, O'Dorisio TM, et al. ${ }^{90}$ Y-DOTATOC dosimetrybased personalized peptide receptor radionuclide therapy. J Nucl Med. 2018;59: 1692-1698.

13. Fendler WP, Reinhardt S, Ilhan H, et al. Preliminary experience with dosimetry, response and patient reported outcome after ${ }^{177}$ Lu-PSMA-617 therapy for metastatic castration-resistant prostate cancer. Oncotarget. 2017;8:3581.

14. Sandström M, Freedman N, Fröss-Baron K, Kahn T, Sundin A. Kidney dosimetry in 777 patients during $177 \mathrm{Lu}$-DOTATATE therapy: aspects on extrapolations and measurement time points. EJNMMI Phys. 2020;7:73.

15. Seo Y, Huh Y, Huang S-Y, et al. Simplified and practical pretherapy tumor dosimetry: a feasibility study for ${ }^{131}$ I-MIBG therapy of neuroblastoma using ${ }^{124}$ I-MIBG PET/CT. Med Phys. 2019;46:2477-2486.

16. Zhao W, Esquinas PL, Frezza A, Hou X, Beauregard J-M, Celler A. Accuracy of kidney dosimetry performed using simplified time activity curve modelling methods: a ${ }^{177}$ Lu-DOTATATE patient study. Phys Med Biol. 2019;64:175006.

17. Jackson PA, Hofman MS, Hicks RJ, Scalzo M, Violet J. Radiation dosimetry in ${ }^{177}$ Lu-PSMA-617 therapy using a single posttreatment SPECT/CT scan: a novel methodology to generate time-and tissue-specific dose factors. J Nucl Med. 2020; 61:1030-1036.

18. Hänscheid H, Lapa C, Buck AK, Lassmann M, Werner RA. Dose mapping after endoradiotherapy with ${ }^{177}$ Lu-DOTATATE/DOTATOC by a single measurement after 4 days. $J$ Nucl Med. 2018;59:75-81.

19. Madsen MT, Menda Y, O'Dorisio TM, O'Dorisio MS. Single time point dose estimate for exponential clearance. Med Phys. 2018;45:2318-2324.

20. Benua RS, Cicale NR, Sonnenberg M, Rawson R. The relation of radioiodine dosimetry to results and complications in the treatment of metastatic thyroid cancer. Am J Roentgenol Radium Ther Nucl Med. 1962;87:171-182.

21. Sgouros G, Song H, Ladenson PW, Wahl RL. Lung toxicity in radioiodine therapy of thyroid carcinoma: development of a dose-rate method and dosimetric implications of the 80-mCi rule. J Nucl Med. 2006;47:1977-1984.

22. Kennedy A, Coldwell D, Sangro B, Wasan H, Salem R. Radioembolization for the treatment of liver tumors: general principles. Am J Clin Oncol. 2012;35:91-99.

23. Strosberg J, El-Haddad G, Wolin E, et al. Phase 3 trial of ${ }^{177}$ Lu-Dotatate for midgut neuroendocrine tumors. N Engl J Med. 2017;376:125-135.

24. Pryma DA, Chin BB, Noto RB, et al. Efficacy and safety of high-specific-activity ${ }^{131}$ I-MIBG therapy in patients with advanced pheochromocytoma or paraganglioma. J Nucl Med. 2019;60:623-630.

25. Quach A, Ji L, Mishra V, et al. Thyroid and hepatic function after high-dose ${ }^{131}$ I-metaiodobenzylguanidine $\left({ }^{131} \mathrm{I}-\mathrm{MIBG}\right)$ therapy for neuroblastoma. Pediatr Blood Cancer. 2011;56:191-201.

26. Hoskin P, Sartor O, O'Sullivan JM, et al. Efficacy and safety of radium-223 dichloride in patients with castration-resistant prostate cancer and symptomatic bone metastases, with or without previous docetaxel use: a prespecified subgroup analysis from the randomised, double-blind, phase 3 ALSYMPCA trial. Lancet Oncol. 2014;15:1397-1406.

27. Sartor O, de Bono J, Chi KN, et al. Lutetium-177-PSMA-617 for metastatic castration-resistant prostate cancer. N Engl J Med. 2021;385:1091-1103.

28. Forrer F, Uusijärvi H, Storch D, Maecke HR, Mueller-Brand J. Treatment with ${ }^{177}$ Lu-DOTATOC of patients with relapse of neuroendocrine tumors after treatment with ${ }^{90}$ Y-DOTATOC. J Nucl Med. 2005;46:1310-1316.

29. Natwa M, Passalaqua S, Chen M-K, et al. Low incidence rates of mucositis, febrile neutropenia or sepsis in the prospective, randomized phase 3 sierra trial for patients with relapsed or refractory acute myeloid leukemia with targeted delivery of anti-CD45 iodine $\left({ }^{131} \mathrm{I}\right)$ apamistamab [Iomab-B] [abstract]. J Nucl Med. 2021;62(suppl 1):1694.

30. Garin E, Rolland Y, Pracht M, et al. High impact of macroaggregated albuminbased tumour dose on response and overall survival in hepatocellular carcinoma patients treated with ${ }^{90} \mathrm{Y}$-loaded glass microsphere radioembolization. Liver Int. 2017;37:101-110.

31. Kappadath SC, Mikell J, Balagopal A, Baladandayuthapani V, Kaseb A, Mahvash A. Hepatocellular carcinoma tumor dose response after 90Y-radioembolization with glass microspheres using 90Y-SPECT/CT-based voxel dosimetry. Int J Radiat Oncol Biol Phys. 2018;102:451-461.

32. Maxon HR, Thomas SR, Hertzberg VS, et al. Relation between effective radiation dose and outcome of radioiodine therapy for thyroid cancer. N Engl J Med. 1983; 309:937-941.

33. Flux GD, Haq M, Chittenden SJ, et al. A dose-effect correlation for radioiodine ablation in differentiated thyroid cancer. Eur J Nucl Med Mol Imaging. 2010;37: $270-275$. 
34. Strom DJ, Thomadsen BR, Suleiman OH, Quinn DM, Miller KL. NCRP Report No. 160, Ionizing Radiation Exposure of the Population of the United States. National Council on Radiation Protection and Measurements (NCRP); 2009.

35. Hänscheid H, Lassmann M, Luster M, Kloos RT, Reiners C. Blood dosimetry from a single measurement of the whole body radioiodine retention in patients with differentiated thyroid carcinoma. Endocr Relat Cancer. 2009;16: 1283-1289.

36. Jentzen W, Bockisch A, Ruhlmann M. Assessment of simplified blood dose protocols for the estimation of the maximum tolerable activity in thyroid cancer patients undergoing radioiodine therapy using ${ }^{124} \mathrm{I}$. J Nucl Med. 2015;56:832-838.

37. Atkins F, Van Nostrand D, Moreau S, Burman K, Wartofsky L. Validation of a simple thyroid cancer dosimetry model based on the fractional whole-body retention at 48 hours post-administration of ${ }^{131} \mathrm{I}$. Thyroid. 2015;25:1347-1350.

38. Chittenden SJ, Pratt BE, Pomeroy K, et al. Optimization of equipment and methodology for whole body activity retention measurements in children undergoing targeted radionuclide therapy. Cancer Biother Radiopharm. 2007;22:243-249.

39. Giammarile F, Chiti A, Lassmann M, Brans B, Flux G. EANM procedure guidelines for $131 \mathrm{I}-m e t a-i o d o b e n z y l g u a n i d i n e ~(131 \mathrm{I}-\mathrm{mIBG})$ therapy. Eur J Nucl Med Mol Imaging. 2008;35:1039-1047.

40. Gustafsson J, Rodeño E, Mínguez P. Feasibility and limitations of quantitative SPECT for ${ }^{223}$ Ra. Phys Med Biol. 2020;65:085012.

41. Ghaly M, Sgouros G, Frey E. Quantitative dual isotope SPECT imaging of the alpha-emitters Th-227 and Ra-223 [abstract]. J Nucl Med. 2019;60(suppl 1):41.

42. Pacilio M, Ventroni G, De Vincentis G, et al. Dosimetry of bone metastases in targeted radionuclide therapy with alpha-emitting 223 Ra-dichloride. Eur J Nucl Med Mol Imaging. 2016;43:21-33.

43. Takahashi A, Miwa K, Sasaki M, Baba S. A Monte Carlo study on ${ }^{223}$ Ra imaging for unsealed radionuclide therapy. Med Phys. 2016;43:2965-2974.
44. Elschot M, Nijsen JF, Lam MG, et al. 99m Tc-MAA overestimates the absorbed dose to the lungs in radioembolization: a quantitative evaluation in patients treated with 166 Ho-microspheres. Eur J Nucl Med Mol Imaging. 2014;41:1965-1975.

45. Dittmann H, Kopp D, Kupferschlaeger J, et al. A prospective study of quantitative SPECT/CT for evaluation of lung shunt fraction before SIRT of liver tumors. J Nucl Med. 2018;59:1366-1372.

46. Lopez B, Mahvash A, Lam MG, Kappadath SC. Calculation of lung mean dose and quantification of error for ${ }^{90} \mathrm{Y}$-microsphere radioembolization using ${ }^{99 m}$ Tc-MAA SPECT/CT and diagnostic chest CT. Med Phys. 2019;46:3929-3940.

47. Hou X, Brosch J, Uribe C, et al. Feasibility of single-time-point dosimetry for radiopharmaceutical therapies. $J$ Nucl Med. 2021;62:1006-1011.

48. Madsen M. Single time point dose estimate for bi-exponential clearance. Soc Nuclear Med; 2017.

49. Bolch WE, Eckerman KF, Sgouros G, Thomas SR. MIRD pamphlet no. 21: a generalized schema for radiopharmaceutical dosimetry - standardization of nomenclature. J Nucl Med. 2009;50:477-484.

50. Dewaraja YK, Frey EC, Sgouros G, et al. MIRD pamphlet no. 23: quantitative SPECT for patient-specific 3-dimensional dosimetry in internal radionuclide therapy. J Nucl Med. 2012;53:1310-1325.

51. Bolch WE, Bouchet LG, Robertson JS, et al. MIRD pamphlet no. 17: the dosimetry of nonuniform activity distributions - radionuclide $\mathrm{S}$ values at the voxel level. J Nucl Med. 1999;40:11S-36S.

52. Siegel JA, Thomas SR, Stubbs JB, et al. MIRD pamphlet no. 16: techniques for quantitative radiopharmaceutical biodistribution data acquisition and analysis for use in human radiation dose estimates. J Nucl Med. 1999;40:37S-61S.

53. Physician Fee Schedule: July 2021 release. https://www.cms.gov/medicaremedicarefee-service-paymentphysicianfeeschedpfs-relative-value-files/rvu21c. Accessed November 1, 2021. 